Revue musicale OICRM

revue musicale oicrm

\title{
Rendre le monde à sa musique. La musique comme puissance d'émerveillement dans le cinéma de Johan van der Keuken
}

\section{Marie Eve Loyez}

Volume 5, numéro 1, 2018

Ce que le cinéma nous apprend de la musique

URI : https://id.erudit.org/iderudit/1044447ar

DOI : https://doi.org/10.7202/1044447ar

Aller au sommaire du numéro

\section{Éditeur(s)}

Observatoire interdisciplinaire de création et recherche en musique (OICRM)

ISSN

2368-7061 (numérique)

Découvrir la revue

Citer cet article

Loyez, M. E. (2018). Rendre le monde à sa musique. La musique comme puissance d'émerveillement dans le cinéma de Johan van der Keuken. Revue musicale OICRM, 5(1), 121-142. https://doi.org/10.7202/1044447ar

\section{Résumé de l'article}

Dans les films de Johan van der Keuken, la musique est tendue entre impulsion et catastrophe : toujours en puissance dans le monde, la musique ne s'actualise pas sans épuiser l'image de ce monde. Par cette tension, le cinéma de van der Keuken nous apprend que la musique peut être au principe d'une redéfinition de la merveille entendue non pas comme objet d'un regard médusant, mais comme production d'une écoute musicienne, corps vibratoire saisi dans son devenir, qui emporte avec lui le monde dans son devenir-musique venant s'y recharger. Cette invention de la musique au cinéma comme puissance d'émerveillement fait de l'oeuvre de van der Keuken le lieu d'une actualisation, à même l'image audio-visuelle et contre les forces réductrices et normalisantes qui la travaillent, d’impératifs éthiques et politiques sur le mode de l'interprétation musicale. 


\title{
Rendre le monde à sa musique. La musique comme puissance d'émerveillement dans le cinéma de Johan van der Keuken
}

\author{
Marie Eve Loyez
}

\begin{abstract}
Résumé
Dans les films de Johan van der Keuken, la musique est tendue entre impulsion et catastrophe : toujours en puissance dans le monde, la musique ne s'actualise pas sans épuiser l'image de ce monde. Par cette tension, le cinéma de van der Keuken nous apprend que la musique peut être au principe d'une redéfinition de la merveille entendue non pas comme objet d'un regard médusant, mais comme production d'une écoute musicienne, corps vibratoire saisi dans son devenir, qui emporte avec lui le monde dans son devenir-musique venant s'y recharger. Cette invention de la musique au cinéma comme puissance d'émerveillement fait de l'œuvre de van der Keuken le lieu d'une actualisation, à même l'image audio-visuelle et contre les forces réductrices et normalisantes qui la travaillent, d'impératifs éthiques et politiques sur le mode de l'interprétation musicale.
\end{abstract}

Mots clés : Johan van der Keuken ; musique ; corps ; merveille ; politique.

\begin{abstract}
The music in Johan van der Keuken's films hangs between impetus and catastrophe: always on the verge of coming into the world, music does not emerge without exhausting the visuality of this world. Through this tension, van der Keuken's cinema tells us that music allows us to redefine the notion of wonders, understood not as objects petrified under Medusa's gaze, but as assemblages that produce a musical hearing. In this understanding, wonders are vibratory bodies captured in the process of their becoming, a process through which the world's becoming-music gets carried on and re-energized. Van der Keuken's films invent a cinematic musical practice that reveals and realizes its potential to evoke wonder, proposing a concrete answer to ethical and political imperatives through the audio-visual image yet against the reductive and normalizing forces at work in it.
\end{abstract}

Keywords: Johan van der Keuken; music; body; wonder; political. 
Une tête d'enfant aveugle s'enlève en plan rapproché sur la fenêtre borgne d'un téléviseur éteint. À l'objet muet, inerte, dont la surface laiteuse occupe les deux tiers du plan, l'enfant posté en marge vole la vedette en prenant sur lui la retranscription de l'événement : une course automobile. De trois quarts, les yeux baissés, légèrement penché en avant, il vrombit dans un microphone, fait jouer ses lèvres, va racler le fond de sa gorge, fait rouler sa langue sur son palais, l'envoie buter contre ses dents - puis il y met les doigts, agace ses lèvres sonnantes, tapote le bout du micro tout en y collant franchement sa bouche hurlante, l'ouvre grand, frôle l'engloutissement - mais déjà tout le corps s'en mêle, par d'amples gestes des bras qui empoignent l'objet à deux mains pour tracer vers ses lèvres toujours vrombissantes, sa langue toujours crissante, des courbes d'approche et d'éloignement - on le croirait à bout de souffle mais non, le micro épuisé est venu se ranger sous une boîte de conserve vide dans laquelle l'enfant s'enfonce à mi-joues pour mieux hurler, râler, racler, grincer, emportant sa déflagration sur une ligne de variation continue de timbres, de résonances, de vibrations, de volumes, d'intensités, imitant à merveille les courbes de vitesse de mille et un bolides lancés plein gaz sur un circuit - ça freine, ça patine, ça dérape, ça accélère, ça s'emballe, un poumon pétarade, une voix se motorise, des dents s'arrachent l'asphalte. Le montage filmique n'est pas en reste qui suit l'affaire en sautant d'échelle à intervalles de plus en plus brefs, tâchant d'emboîter les vitesses, les fulgurances, les improvisations, les désynchronisations - plan rapproché, gros plan, plan large et ainsi de suite, changement d'axe soudain pour prendre pleine face les jeux de bras dans toute leur assurance, gros plan sur la boîte qui avale l'enfant qui avale la boîte, décadrage discret sur l'oreille tandis que langue et micro s'agitent de plus belle dans le coin inférieur gauche... (Herman Slobbe, l'enfant aveugle 2 (1966), extrait vidéo 1$)$.

Extrait vidéo 1: Johan van der Keuken, Herman Slobbe, l'enfant aveugle 2 (1966), Herman rejoue la course automobile, 18:10-19:20 (C) Arte France Développement, Idéale Audience International, Pieter van Huystee Film, 2007.

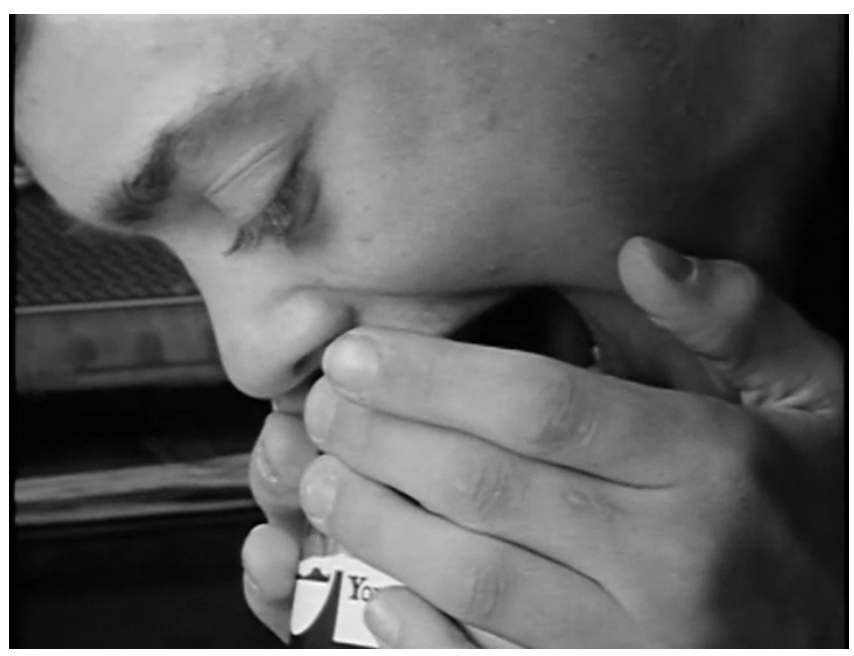

Qu'est-ce qui s'est passé ? Herman, quatorze ans, aveugle de naissance, s'est laissé traîner de mauvais gré à une course automobile, et le film lui met dans les mains la chance d'en faire son événement : la création d'une pièce vocale originale, qui a ceci pour nous de saisissant qu'elle met en jeu, d'un même tenant et durant tout le temps de son exécution, la musique à la fois dans son impulsion et dans sa catastrophe-comme impulsion, comme catastrophe. Deux temps de la musique que nous interrogerons tout au long du texte 
dans leur capacité à rendre quelque chose d'un tempo saisi à même le monde, suivant des modalités de composition audio-visuelle qui vont traquant, révélant, exploitant, épuisant, rechargeant les puissances de la musique. Car pour Herman, celle-ci est une question qu'il ne cesse de poser, en reniant sa version pulmonaire d'Hello Dolly que l'on entend pourtant dès le générique d'ouverture du film ; en s'improvisant à tout bout de champ critique (sévère) des derniers tubes à la mode ou animateur radio pour les plantes vertes du salon; en fendant de son harmonica cadence et harmonie pour y faire déborder par le milieu une matière sonore proliférante. Mais lorsqu'il s'abouche avec un microphone et une boîte en fer-blanc pour rejouer sur un mode musical une course automobile, il produit une réponse triple qui relance aussitôt, chez van der Keuken, une triple question que nous tâcherons de relayer pour en déplier les implications : la musique est en puissance - comment capter et rendre perceptible la musique à l'œuvre dans l'existence ? La musique est une puissance - que peut la musique pour le cinéma documentaire? La musique est une puissance paradoxale dont le mode d'effectuation est d'épuisement - en quoi l'épuisement peut-il s'agencer en un protocole d'expérimentation audio-visuelle d'un problème de cinéma documentaire que l'on pourrait formuler dans les termes de Vers le sud (1981) : «toucher le réel »?

«ENTENDRE L'ARBRE ${ }^{1} »:$ LA MUSIQUE EN PUISSANCE

Que la musique soit toujours en puissance, la tâche pourrait bien revenir au cinéma de le montrer. Prenez un petit gars mal embouché, emmenez-le de force au Grand Prix, et il vous en extirpe une composition inouie en machinant sa voix avec ce qu'il a sous la main : ses doigts, ses lèvres, une tête de micro, la gueule béante d'une vieille conserve. La musique n'avait jamais cessé d'être en devenir, l'enfant dans toute sa candeur n'a pas cru qu'il fût nécessaire de se saisir d'instruments complexes ni de noircir une partition pour donner l'impulsion à son actualisation. En revanche, il aura fallu être là pour le filmer, mettre le micro sur son chemin, saisir en image autant qu'en son tous ces gestes de bras et de gorge qui s'arrachent un chant de culasses et de virages à partir de sensations fortes et de trois bouts de ficelle. Prodigieuse performance que celle de ce corps curieusement appareillé dont le film met au jour l'écoute proprement musicienne, seule à même de produire une imitation qui excède son modèle sans le trahir ! Car c'est ainsi que peut s'entendre la musicalisation de l'événement par Herman : la révélation, par la mise en série des tenseurs et des tensions (changements de timbres, exploration des modalités vibratoires de la langue, des lèvres, des dents, avec ou sans les doigts, recherche de toute une gamme de résonances et de volumes par des variations de posture ou l'invention d'une combinatoire de membres et d'objets...), des différences sonores perçues dans leurs moindres nuances pendant la course et que la créativité de l'enfant démultiplie à plaisir. Sans doute fallait-il encore toute la dextérité d'un montage pour montrer, moins

1 Dans L'enfant aveugle (1964), une jeune fille s'indigne de l'injustice des voyants qui considèrent les aveugles comme déficients alors qu'eux-mêmes sont incapables « d'entendre l'arbre ». 
l'efficace ébouriffante de l'imitation des bolides², que la manière dont la composition sonore finit par s'autonomiser de sa source d'inspiration pour gagner une puissance rythmique, corporelle, vibratoire, détachée de tout référent extérieur ; mais aussi pour recomposer une trajectoire expérimentale qui part d'un essai de voix seule modulant des accents à la Louis Armstrong, passe par l'instrument harmonica et ses potentiels de déstructuration, pour en arriver enfin à l'invention propre d'une instrumentation de la voix, improvisant ses amplificateurs et modulateurs d'effets, créant un complexe voix/doigts/bouche/boîte/micro/langue/oreille/genoux traversé d'une ligne de course vociférante et d'une mauvaise humeur jazzée d'adolescent, qui remodèle les profondeurs de gorge d'Armstrong et reconfigure les manipulations familières au joueur d'harmonica, les retourne, les adapte, les développe, les métamorphose au contact d'objets qui, détournés eux aussi, mal tenus malmenés, deviennent tout autre chose - caisse de résonance, boîte de Pandore. Car à la limite, ce déferlement emporte dans son sillage jusqu'au joyeux concours d'insultes morbides ("Pustules ! cancer ! typhus! tuberculose ! choléra! peste ! ...let's go!») auquel Herman se livre avec ses amis scouts, et qui finit par sonner comme un autre mouvement - catastrophique s'il en est : une joyeuse décharge, digne des envolées cathartiques d'un Capitaine Haddock, de tous les maux du monde ! - d'une existence dont le moindre geste est en instance de musique.

On ne pensera pas que la musique ne sait plus chanter, dans un monde devenu mécanique ou atomique, mais plutôt qu'un immense coefficient de variation affecte et entraîne toutes les parties phatiques, aphatiques, linguistiques, poétiques, instrumentales, musicales d'un même agencement sonore - " un simple hurlement parcourant tous les degrés » (Th. Mann) (Deleuze et Guattari 1980, p. 122).

Impulsion, catastrophe : chez van der Keuken, la musique se rappelle à nous non comme système complexe, comme technique ou comme virtuosité, mais comme un tenseur qui s'épuise à faire claquer ses limites, à (se) tendre vers tout ce qu'il n'est pas encore, mais toujours déjà en puissance, à (se) tendre vers ce qu'il n'est déjà presque plus, mais ne demande qu'à être rechargé ou saisi dans son épuisement même. Ainsi de ces innombrables passages, au long de la filmographie, où la musique est une course de relais de voix qui s'abîment dans la psalmodie (Vacances prolongées (2000), extrait vidéo 2), la monodie grinçante de deux tasses entrechoquées (ibid., extrait vidéo 3), l'essoufflement mélancolique d'un saxophone qui se perd dans un flou de barges sur l'Amstel (ibid.), l'éraillement d'un rire scandant des poèmes de deuil (Bert Schierbeek, la porte (1973), extrait vidéo 4), les gargouillements intestinaux d'un bombardon (Cuivres débridés, à la rencontre du swing (1993), extrait vidéo 5), une brise andine s'élevant d'une flûte dans un appartement d'Amsterdam (Amsterdam Global Village (1998), extrait vidéo 6)...

2 Van der Keuken fait d'abord alterner des plans sur Herman assistant à la course avec des plans des voitures lancées sur le circuit, qu'il mixe subrepticement avec la recréation sonore du garçon - provoquant alors la surprise chez le spectateur lorsqu'arrive la série de plans décrite plus haut, qui révèle la machination de la bande sonore de la course et fait prendre la mesure du talent d'Herman. 


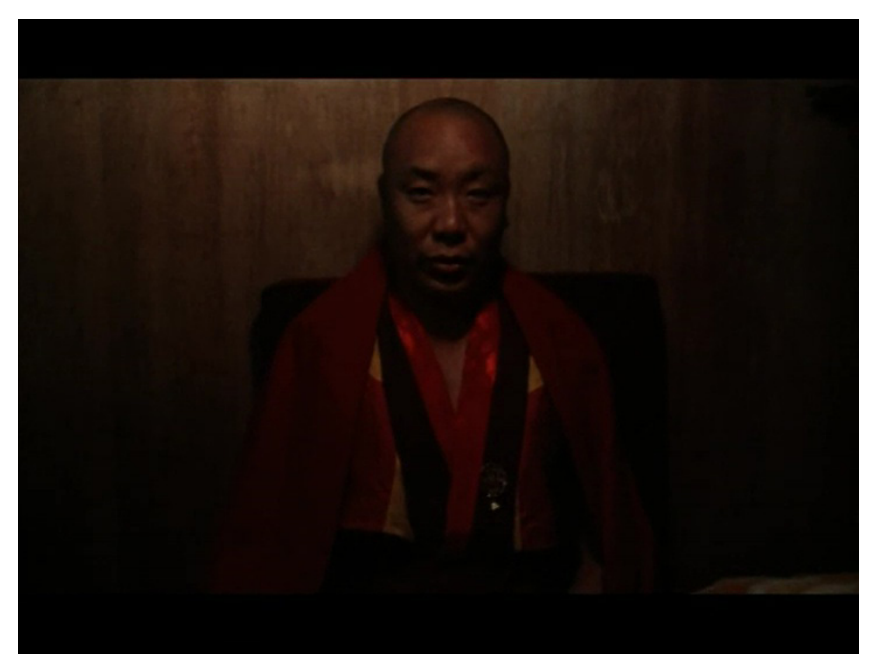

Extrait vidéo 2 : Johan van der Keuken, Vacances prolongées (2000), Continuum des ablutions à la récitation d'un moine bouddhiste, 21:30-22:45 (C) Arte France Développement, Idéale Audience International, Pieter van Huystee Film, 2007.

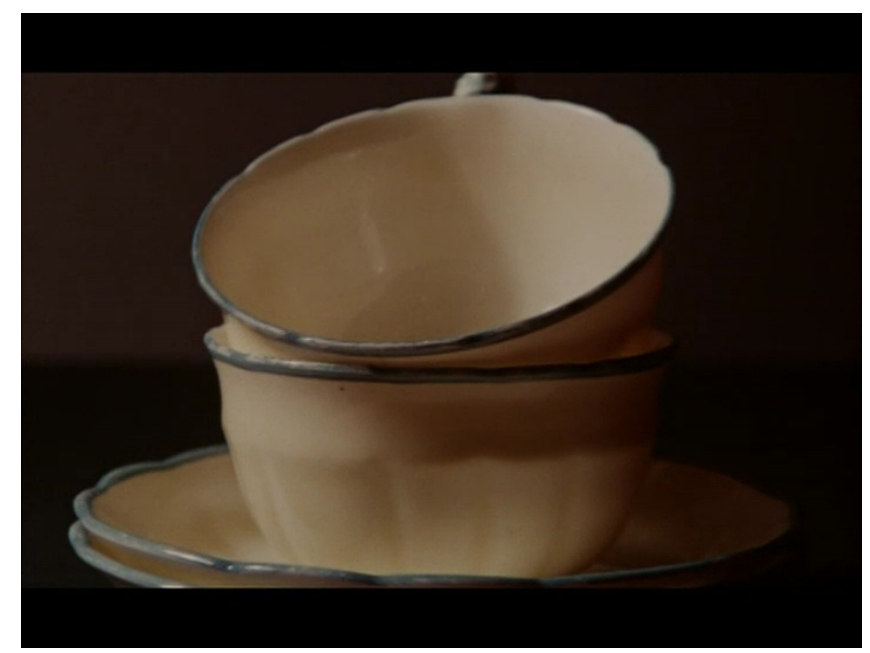

Extrait vidéo 3 : Johan van der Keuken, Vacances prolongées, Tremblement de tasses, 00:00-01:14 (C) Arte France Développement, Idéale Audience International, Pieter van Huystee Film, 2007.

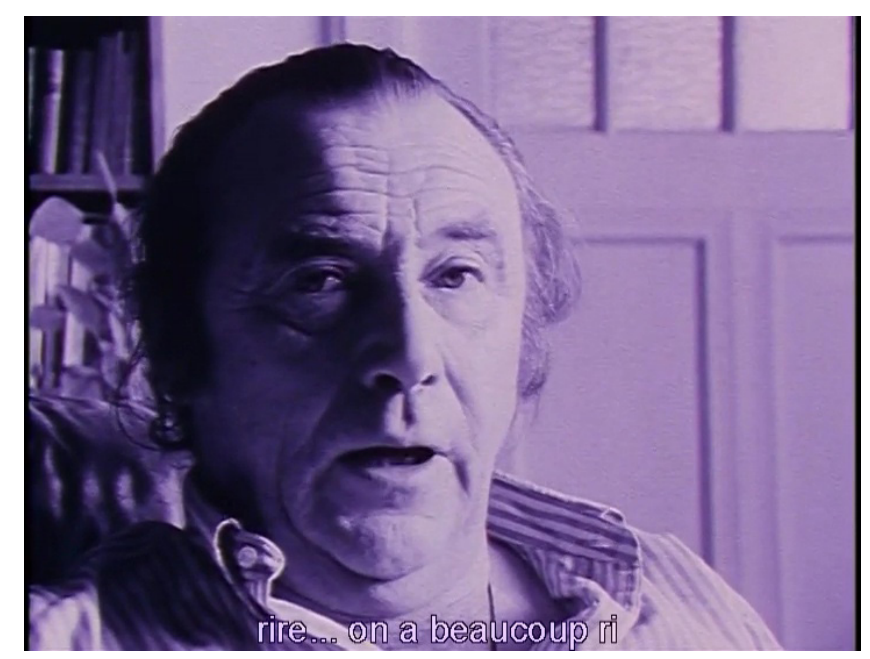

Extrait vidéo 4 : Johan van der Keuken, Bert Schierbeek, la porte (1973), Le rire du poète,

04:04-05:09 @ Arte France Développement, Idéale Audience International, Pieter van Huystee Film, 2007. 
Extrait vidéo 5 : Johan van der Keuken, Cuivres débridés, à la rencontre du swing (1993), Si bien qu'on ne sait plus qui du filmeur, qui du bombardon..., 37:40-38:37 (C) Arte France Développement, Idéale Audience International, Pieter van Huystee Film, 2006.
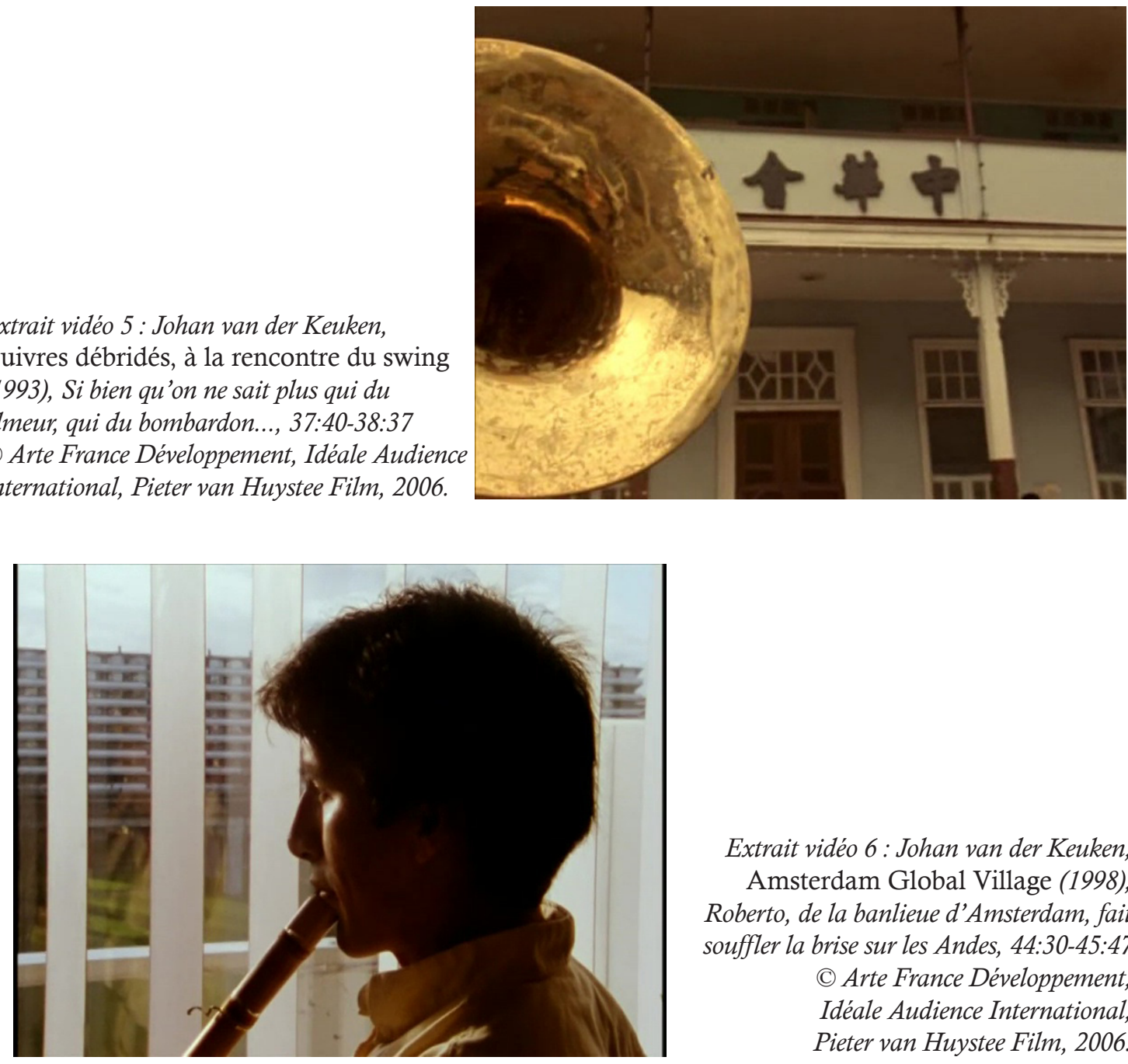

Extrait vidéo 6: Johan van der Keuken, Amsterdam Global Village (1998), Roberto, de la banlieue d'Amsterdam, fait souffler la brise sur les Andes, 44:30-45:47 (C) Arte France Développement, Idéale Audience International, Pieter van Huystee Film, 2006.

Le cinéma serait du reste d'autant plus voué à rejouer et rendre perceptible la musicalité de l'être ou de l'événement qu'il est, selon les mots d'Alain Badiou, l'art du contemporain, c'est-à-dire le seul qui compose littéralement avec «l'imagerie contemporaine » (Badiou 2010, p. 370) - a fortiori, ajouterions-nous, le cinéma documentaire :

Le matériau est commun à tous les films, donc tout le monde peut venir, tout le monde s'y reconnaîtra. Le cinéma peut reproduire le bruit du monde ; il peut aussi inventer un nouveau silence. Il peut reproduire notre agitation ; il peut inventer de nouvelles formes d'immobilité. [...] Mais, au départ, les matériaux sont les mêmes, et c'est la raison pour laquelle des millions de gens peuvent juger un film comme étant contemporain de leur existence, alors qu'ils ne le peuvent pour les autres arts qu'avec une longue éducation (ibid.).

En cette qualité d'« art de masse » (ibid., p. 331), « le cinéma retient de la musique [...] une certaine dialectique du visible et de l'audible, donc le charme du son lorsqu'il est placé dans l'existence. » (ibid., p. 336). Il nous semble que le cinéma de van der Keuken, par sa pratique serrée du tissage audio-visuel, se rend particulièrement propice au surgissement d'une musicalité repérée à même le monde - le « contemporain »-, " comme une musique sans musique, une musique sans technique musicale, une musique 
empruntée puis redonnée à l'existence " (ibid.). Si l'improvisation sonore d'Herman est d'abord entendue montée sur les images de la course, c'est peut-être pour que s'opère dans l'après-coup de la surprise la mise en évidence d'une musicalité de fait, dépassant la composante strictement acoustique, des folles ellipses comme des décrochages incontrôlés des bolides. Le film nous pousse, par un apprentissage expérimental de l'écoute, à saisir l'événement comme musique.

Cuivres débridés, à la rencontre du swing propose de ce point de vue une ouverture magistrale. C'est une rumeur qui nous arrive en premier, précédant les images, précédant même le générique ${ }^{3}$, comme toute rumeur toujours précède tout ce qui arrive, précède le monde - ou le fait naître ? Une rumeur matinale fait naître dans sa brume d'oiseaux, de voix et de cloches des images avant même les images : on imagine un matin de montagne, d'herbe et d'eau pure, de pâturages en altitude. Apparaît alors un paysage en devenir : au lointain d'un pâle contre-jour, une petite silhouette chargée d'on ne sait quel fardeau dévale par la droite à petits pas sautillants le flanc sec, maigrement buissonneux d'une montagne qui fend dans sa diagonale l'écran de brume en arrière-plan, d'où peinent à transpercer les courbes encore sommeillantes d'une vallée. Une coupe reprend le personnage un peu plus bas, en pied et de profil, traversant le cadre de droite à gauche ; une autre le laisse pour aller l'attendre à l'étage inférieur où il surgit bientôt toujours par la droite, avançant au pas de l'habitude, ne laissant plus de doute sur ce qu'il porte sur le dos : une machine à coudre ; la caméra le prend maintenant de trois quarts en plongée légère, couvrant doucement sa descente devenue plus prudente sur un raidillon, le suivant de dos lorsqu'il la dépasse, élargissant le cadre pour mieux saisir, tandis qu'il poursuit sa route, les lignes dynamiques des contreforts de la montagne, celles de la vallée vers laquelle il tend. Tout au long de son trajet, nous avons pu voir autour la brume lentement se dissiper, dévoiler peu à peu le champ d'intensités des verts, des jaunes, des ondulations de cultures en terrasses. Peu à peu, une autre rumeur s'est superposée à la première, celle d'un vacarme encore lointain de cuivres et de percussions, qui enfle sur le dernier plan d'extérieur où l'on voit notre homme pénétrer dans un bâtiment de terre crue - pour atteindre son plein volume à l'intérieur, où deux femmes manipulent des machines à coudre d'où semblent provenir, non seulement les roulements des manivelles et le martèlement des aiguilles mécaniques, mais l'ensemble de la musique. L'impression persiste alors que la caméra s'arrête en plan large sur ces agencements corps-machines dont les mouvements fluides et énergiques génèrent, de fait, une rythmique invitant au développement, et plus encore lorsqu'un gros plan sur les mains et l'aiguille affairées accuse la synchronisation de ce fond de basse avec le déchaînement de cuivres qui envahit l'espace. Un plan en plongée sur les femmes aux machines se déploie alors en travelling ascendant et nous emporte vers l'étage supérieur, où toute une troupe de musiciens de tous âges tape et souffle dans toutes sortes d'instruments de cuivre et de peau tendue, dans un mélange ahurissant de contrôle et d'affolements. Mais ce 
n'est que lorsque la caméra se dirige vers la fenêtre pour capter le geste de l'un des pavillons qui semble se charger à lui seul de faire rejaillir toute la musique au dehors, ce n'est que lorsque la caméra accompagne au dehors la musique se déversant sur la montagne qui semble alors faire serpenter ses terrasses et résonner ses couleurs de concert, que l'on peut saisir où est la musique dans cette séquence. La musique n'est pas - n'est pas seulement - l'ensemble de sons produits par les musiciens à l'étage du bâtiment de terre crue. La musique est, au sein de la relation audio-visuelle, le tenseur de l'image, celui qui l'épuise de l'intérieur pour en faire ressortir toutes les lignes de crête et les lignes d'horizon, les ombres dansantes, la cambrure de ses layons ; pour rendre perceptible, dans la résonance de ses impulsions - une cloche s'agite, une voix s'élève, une pente se dévale, une manivelle se tourne, deux cymbales se percutent et de ses catastrophes - la cloche s'immobilise, une toux emporte la voix, une main se fatigue, une vibration se perd dans le tracé d'un sillon -, l'événement avec lequel elle tend à se confondre, car c'est elle qui le recompose sur le champ de force de ses intensités. Qu'est-ce qui s'est passé ? Une rumeur matinale fendue de diagonales herbeuses a dévalé une montagne en l'emportant sur le dos d'une machine à coudre, s'est enflée de brume pour mieux la dissiper en faisant ondoyer les rizières, est entrée dans une ronde de mains et de manivelles, s'est ramifiée pour traverser mille trachées métalliques, avant de se ramasser toute pour expirer à nouveau sur la montagne en ridant ses flancs : « une musique [a été] empruntée puis redonnée à l'existence »(ibid.).

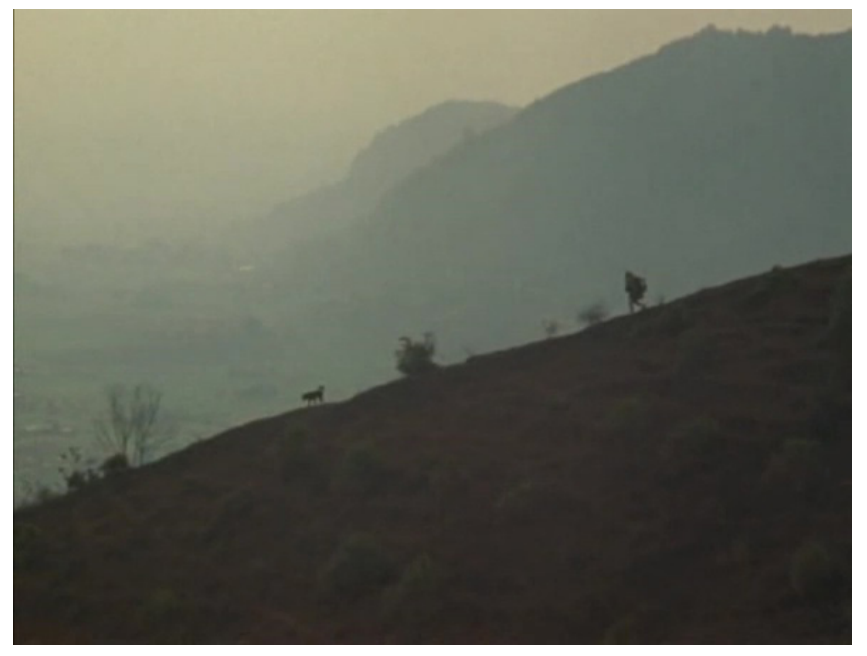

Extrait vidéo 7 : Johan van der Keuken, Cuivres débridés, à la rencontre du swing, "Une musique empruntée puis redonnée à l'existence", 00:00-03:50 (C) Arte France Développement, Idéale Audience International, Pieter van Huystee Film, 2006.

\section{TOUCHER L'OISEAU : LA MUSIQUE COMME PUISSANCE}

Aussi le cinéma nous montrant que la musique est en puissance, nous apprend-t-il du même élan qu'elle est elle-même une puissance. Car épuiser l'image en la travaillant de l'intérieur, c'est-à-dire depuis la relation audio-visuelle dont elle traverse toutes les composantes, équivaut non pas à détruire l'image ou la porter à son point d'abolition, mais, comme nous avons tenté de le décrire, à la mettre en tension, c'est-à-dire la 
forcer à rendre tout son potentiel. C'est à ce titre que la musique nous semble jouer un rôle essentiel pour un cinéaste qui s'efforce de «toucher le réel », a fortiori lorsqu'il pense et le réel et l'image « en termes énergétiques »:

Je n'envisage pas la réalité comme quelque chose qui puisse être fixé sur la pellicule mais plutôt comme un champ (en termes énergétiques). C'est peut-être vague. Ce que je veux dire c'est que l'image filmée, telle que j'essaie de la faire, résulte plutôt d'une collision entre le champ du réel et l'énergie que je mets à l'explorer (Van der Keuken 1998, p. 14).

À la fin de Vers le sud, au terme d'un voyage qui l'a mené d'Amsterdam au Caire en passant par plusieurs régions de France et d'Italie, van der Keuken repère une façade d'échoppe dont un pan de mur est couvert d'un motif géométrique peint en trompe-l'œil. Il zoome lentement jusqu'à ce que l'image se confonde totalement avec la peinture et fait ce constat : "De retour dans la ville, je me dis : c'est difficile, de toucher le réel. " À ces mots, sa main entre dans le champ et va effleurer le mur peint (extrait vidéo 8).

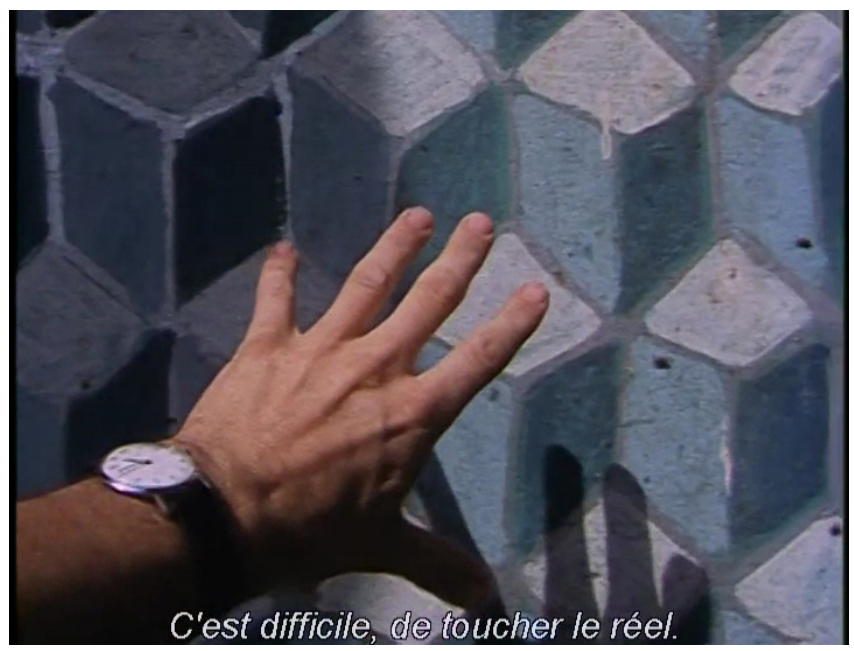

Extrait vidéo 8: Johan van der Keuken, Vers le sud (1981), "C'est difficile, de toucher le réel », 02:20:3202:21:28 (C) Arte France Développement, Idéale Audience International, Pieter van Huystee Film, 2007.

La force de ce passage tient dans sa littéralité, d'autant plus frappante qu'elle trouve en 1981 la formule d'un problème soulevé quinze ans auparavant par et dans la seule image, à l'occasion de l'expérience liminaire des films sur les enfants aveugles, problème sans cesse relancé ensuite d'un film à l'autre. Dans une séquence de L'enfant aveugle, le réel en question est un oiseau. Or, celui-ci passe sans doute quelque part entre le modèle empaillé aux plumes défraîchies, raide sur son socle, sur lequel une fillette non-voyante promène consciencieusement la main sans s'émouvoir (s'arrêtant sur la bille de verre dont la dureté, la fixité et la froideur font si mal sentir ce que peut être un œil) ; et la petite masse vivante, chaude et soyeuse, informe, prenant mille formes tant elle s'agite et se défait, se déploie soudain dans un frottement par le côté, le cour battant d'affolement dans les mains de la petite fille qui rit d'aise de sentir une aile éventer sa joue et de pouvoir enfin toucher l'oiseau. Toucher l'image, fût-elle emplumée et en trois dimensions, n'est rien moins que toucher le réel - c'est que 
l'on n'est pas sorti d'un système d'apprentissage fondé sur la toute-puissance de la représentation et du langage, et sur le principe de leur coïncidence. Toucher un oiseau empaillé, puis le mot en braille correspondant, équivaut à l'expérience du petit écolier bien-voyant à qui l'on apprend à réciter le plus vite possible le mot " oiseau » sur l'image d'un pigeon dans La leçon de lecture (1973). Or, ce dernier film s'emploie vigoureusement à défaire cette méthode au moyen d'un montage audio-visuel impliquant la célèbre pièce électronique Popcorn composée par Gershon Kingsley en 1968, qui joue de l'inquiétude subtile d'un thème répétitif, à la fois par des lignes de basse jouant le rôle de variables aléatoires, et par le simple mais génial effet de suggestion du titre qui insinue dans la régularité du tempo les sautes imprévisibles de grains de maïs éclatant sur le feu. La musique entraîne alors l'image dans son rythme effréné jusqu'à lui faire rebattre ses propres cartes : le montage calquant initialement les associations pédagogiques mots-illustrations et la monotonie de la récitation, semble perdre le contrôle sous l'effet d'une musique extra-diégétique qui creuse progressivement son chemin dans l'image, images et sons s'emportant mutuellement dans un emballement partagé, jusqu'à créer de nouvelles associations et revitaliser - débrider - le rythme proprement musical en latence dans la récitation. Qu'est-ce qui s'est passé? Une musique artificielle, hétérogène au film tel qu'il fut tourné, a été " placé[e] dans l'existence » (Badiou 2010, p. 336), au travers d'un montage audio-visuel organisant tous ses éléments sonores (extra-diégétiques et diégétiques ; bruits, musique, voix...) et visuels sur un même plan intensif ; emportant dans son sillon la récitation monotone d'associations régulières entre mots et images, elle en a épuisé l'arbitraire et rechargé le potentiel poétique, mettant en branle le système éducatif hollandais. La musique portait en elle cette puissance ; l'image en l'accueillant 1'a non seulement actualisée, mais rechargée elle aussi en un geste réciproque, en lui conférant une portée politique que seul le contexte du film, et le montage " actif, agressif " (Van der Keuken 1998, p. 14) de van der Keuken pouvaient générer. "Toucher le réel » a bien ici la puissance d'une commotion, suivant une terminologie familière au cinéaste qui reconnaît la "force de frappe de la caméra » (ibid., p. 184).

Mais revenons à la séquence de l'oiseau dans L'enfant aveugle, qui permet de saisir combien l'effort continu de van der Keuken pour faire tendre l'image vers un toucher doit s'entendre de la façon la plus littérale. Le problème du cinéaste n'est pas ici de toucher l'oiseau, mais de toucher «toucher-l'oiseau ", autrement dit de reproduire de faire faire au spectateur au travers de l'image audio-visuelle - l'expérience sensible d'une jeune aveugle tenant entre ses mains un oiseau. C'est encore la musique qui nous semble le vecteur essentiel de la transposition d'un tact qui devient un tact de l'écoute. Lorsque l'on passe du plan sur la fillette parcourant la surface de l'animal empaillé, à ceux où elle embrasse et caresse l'animal vivant, s'élève une valse mélancolique très simple jouée au piano, avec une application un peu poussive et quelques hésitations : celles-ci ramènent sur le plan sonore les mains du pianiste, qui se rappellent à nous dans la matérialité et la gestualité de leur toucher par les tâtonnements qui brisent tout élan d'abstraction de la musique - ou plutôt de l'écoute, car c'est un certain type d'écoute qui s'attache à sublimer la musique, feignant d'oublier qu'elle a un corps. En l'occurrence, la petite valse se retrouvant prise dans la relation audio-visuelle 
compose si bien avec l'image que le toucher délicat, incertain, trébuchant du pianiste vient comme affleurer dans les mains tendres, timides, légèrement inquiètes de la fillette $^{4}$ (extrait vidéo 9). Les ratés et les reprises, la persévérance et l'émotion de l'interprétation s'entendront mieux encore lorsque la petite valse sera remontée sur un plus long passage lors de la sortie de deux autres jeunes filles, livrées à elles-mêmes avec leur seule canne blanche et leurs petits escarpins sur un trottoir plein d'embûches. L'analyse de la scène de l'oiseau est indissociable de cette écoute répétée du morceau de musique à différents moments du film, qui renforce, dans ses petits degrés de différence et par une forme d'impact rétrospectif, notre perception de la fragilité de chacun d'eux (extrait vidéo 10). Manipulation émue et un peu gauche d'un animal épeuré ; exécution maladroite et expressive d'une mélodie mélancolique : ce qui se joue dans cette combinaison où chacun (valse, fillette, oiseau, caméra, auditeurspectateur) gagne un corps, est l'interprétation de l'image par la musique - celle-ci ne glissant pas sur la surface de l'image comme la main restée de marbre d'une fillette sur la représentation empaillée d'un oiseau, mais passant juste dessous la peau de l'image pour y faire monter le musical, comme la caresse d'une fillette, insinuant sa joie de peur mêlée par la pression de ses doigts sous l'échappée d'une aile, fait saillir le petit cœur battant d'une colombe. Dans le cinéma de van der Keuken, la musique est bel et bien « ce corps qui bat $»^{5}$.

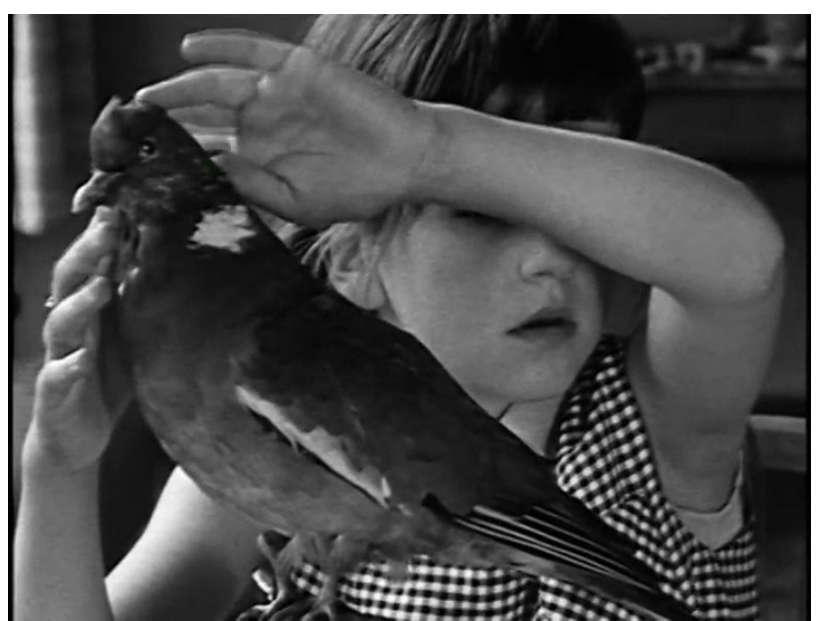

Extrait vidéo 9: Johan van der Keuken, L'enfant aveugle (1964), Toucher l'oiseau, 07:23-08:04 (C) Arte France Développement, Idéale Audience International, Pieter van Huystee Film, 2007.

4 On retrouve un procédé similaire dans Herman Slobbe, l'enfant aveugle 2, lorsque Le matin des Noirs d'Archie Shepp emboîte le pas des scouts non-voyants en prêtant à leur marche à la fois volontaire et accidentée - impulsions, catastrophes -, en léger contretemps comme si venait se loger dans l'infime intervalle le temps nécessaire à l'interprétation, les errements tortueux, les achoppements, les déraillements du saxophone - ses essoufflements, mais aussi sa manière de tenir dans l'exténuation, ses sursauts et ressauts d'après les chutes - tandis qu'il fait composer ses méandres avec la constance et la brillance du vibraphone, scansion régulière, surface étale qui aide à sortir de l'ornière, aplanissement d'un terrain dont le saxophone retrace les embûches, trébuchent les aveugles ils gardent belle allure, scouts toujours (extrait vidéo 11).

5 «Dans les Kreisleriana de Schumann, je n'entends à vrai dire aucune note, aucun thème, aucun dessin, aucune grammaire, aucun sens, rien de ce qui permettrait de reconstituer quelque structure intelligible de l'oeuvre. Non, ce que j'entends, ce sont des coups : j'entends ce qui bat dans le corps, ce qui bat le corps, ou mieux : ce corps qui bat » (Barthes 1975, p. 217). 


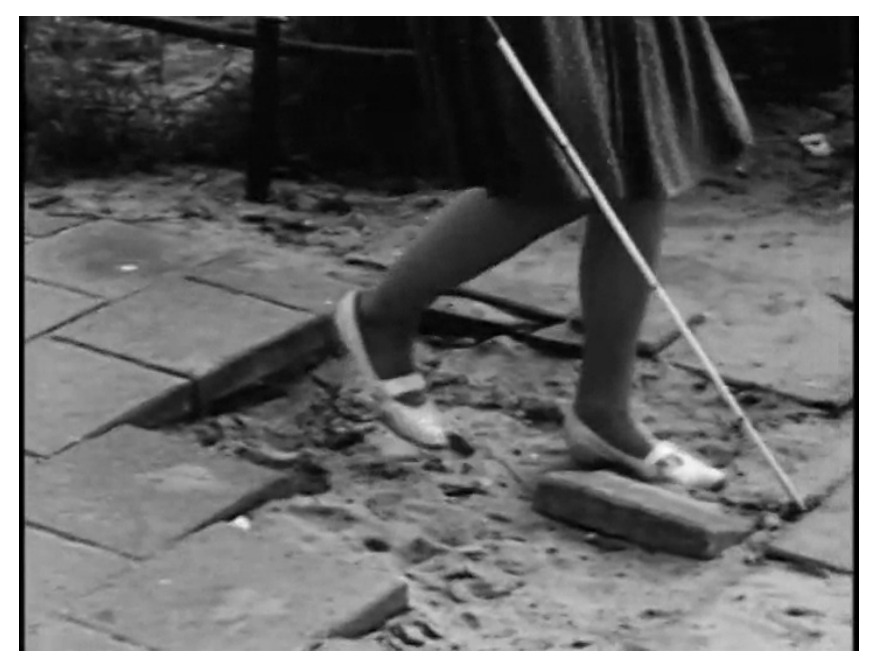

Extrait vidéo 10 : Johan van der Keuken, L'enfant aveugle, Deux jeunes filles aveugles apprennent à se repérer sur un territoire qui les exclut de fait, 19:35-20:51 (C) Arte France Développement, Idéale Audience International, Pieter van Huystee Film, 2007.

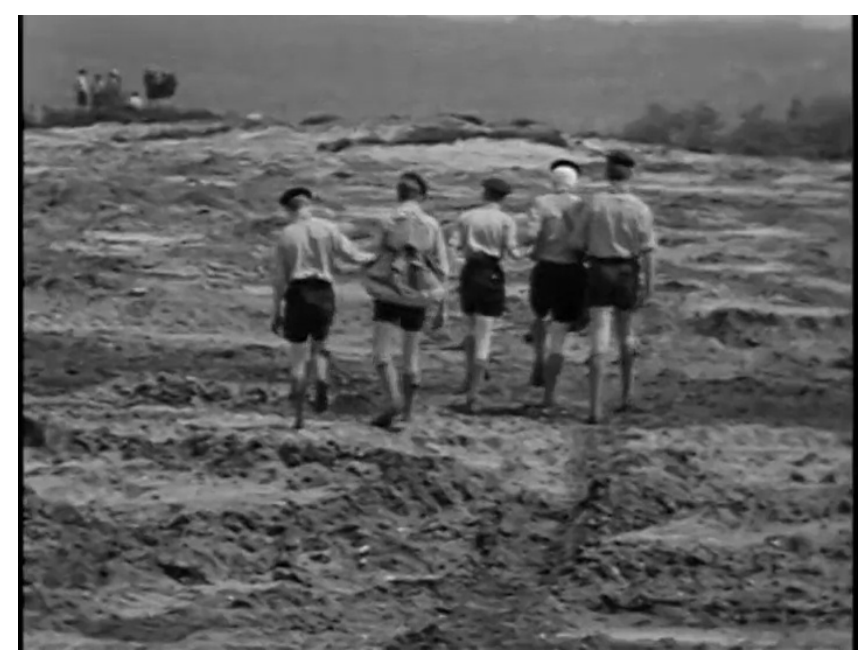

Extrait vidéo 11 : Johan van der Keuken, Herman Slobbe, 1'enfant aveugle 2, Scouts toujours, 12:00-13:05 (c) Arte France Développement, Idéale Audience International, Pieter van Huystee Film, 2007.

\section{DANSER L'ÉLÉPHANT : LES PUISSANCES ÉTHIQUES ET POLITIQUES DE LA MUSIQUE}

Ce modelage, dans la relation audio-visuelle, d'un tact de l'écoute ébauché sur une préhension du monde par les aveugles qui relève d'une forme d'interprétation musicale (entendre l'arbre, toucher l'oiseau, vocaliser une course automobile), nous paraît faire lever chez van der Keuken une gestualité propre répondant à une méfiance douloureuse à l'égard de l'image, dont il respecte et entend bien par ailleurs exprimer le formidable potentiel. L'idée d'épuisement ne nous semble pas étrangère à la démarche documentaire, qu'elle rencontre précisément dans son désir d'exhaustivité, lui-même impliqué dans la tendance d'une culture foncièrement visuelle à tout mettre en image, et qui pourrait trouver une formulation possible dans une série de procédures ou de mots d'ordre - parcourir le monde, le couvrir, en tenir le compte, le répertorier, le 
classifier, le décalquer, le comparer, l'élucider... avec tout ce que cela peut entraîner de mises en boîte et en vitrine, de clichés mortifères, de têtes réduites et de têtes coupées. Autrement dit, à cette double injonction a priori contradictoire : d'un côté, reconnaître dans l'image une voie d'accès à une expérience du réel, de l'autre, traquer sans relâche les dictats sociaux, idéologiques, politiques dont l'image est un véhicule privilégié, le cinéaste répond par une cinématographie de la cécité volontaire, où il n'est pas question de fermer les yeux ni de renoncer à l'image, bien au contraire, mais de s'ouvrir à un type d'écoute enseigné par l'expérience perceptive des aveugles. Il s'agit alors de provoquer un double phénomène de dépense et de compensation des composantes visuelles de l'image cinématographique, une sorte de redistribution de ses forces contraignantes et de ses forces libératoires. La tension de l'image par la musique - épuisant le cliché (dans toute sa servilité complaisante à l'égard du pouvoir et de ses normes), et repotentialisant d'autant la part maudite (tout ce que le pouvoir normalisant juge indigne de montrer, et renvoie constamment dans les marges de l'indécence, de la barbarie ou de l'anormalité) - en révèle, en accuse, en renverse les enjeux éthiques et politiques. C'est jouer, en somme, l'exhaustion de l'image comme processus illimité, comme ouvroir d'interprétation potentielle, contre l'exhaustivité comme achèvement ou comme point final ; et à travers elles, la mise en rayonnement de la singularité de l'événement contre la définition bornée d'une généralité de l'être. Six ou sept éléphants suffiront à nous en convaincre. Comment restituer l'émerveillement suscité chez le voyageur par le surgissement d'une colonne d'éléphants au milieu de la rue sans sacrifier à l'exotisme, sans négliger de montrer dans le même temps comment une telle procession s'intègre dans le quotidien d'une ville indienne? Comment agencer la conjonction des points de vue ou des expériences - celle du cinéaste et des spectateurs occidentaux d'un côté, de l'autre celle de la population locale dont le film prétend "toucher le réel »- pour rendre dans toute sa complexité un événement qui est, dans le même temps et sous toutes ses faces, à la fois surprise et habitude, rupture et continuité, à la fois d'une beauté à couper le souffle et pris dans la trame érodée des travaux et des jours? Dans cette séquence de L'œil au-dessus du puits (1988, extrait vidéo 12), van der Keuken choisit de faire composer un cadrage et une musique qui tout à la fois préparent une scène, dramatisent une apparition, et rendent un sentiment de familiarité. Ainsi, un plan large pris en plongée depuis un avant-toit sur l'activité diffuse de la rue qui se fend pour laisser une zone de passage, s'institue comme cadre et comme fond, anticipe ou appelle l'arrivée des éléphants par le coin supérieur droit. La musique indienne qui joue sur des sons tenus et des silences, un tempo lent soudain accéléré, une respiration suspendue dans les vibrations d'un sitar, fraye le chemin aux lourdes bêtes qui se balanceront de leur démarche nonchalante, à la fois élégante et pesante, scandée comme par une horloge massive par leur trompe en balancier. Par ce double geste de composition, un événement vient d'être signalé et son aura magnifiée. Pour autant, parce que le cinéaste a choisi de monter de tels plans urbains foisonnants plus tôt dans le film, et surtout parce que la musique a déjà été entendue plusieurs fois, épuisant son propre effet d'exotisme, celui des éléphants 
se trouve désamorcé ${ }^{6}$ : l'événement est dans le même temps rendu à l'évidence de son surgissement dans un tel contexte. Il faut encore relever, dans l'impulsion donnée par la musique extra-diégétique, la composition proprement musicale des plans par un cadrage qui à la fois souligne la beauté exceptionnelle de la scène en en faisant ressortir les jeux de roues et de hanches, les ondulations réciproques, les perspectives contrariées, les chassés-croisés de chair et de taule (dans le premier plan, le flot des piétons, puis celui des cyclistes, la colonne enfin des voiturettes à trois roues, toutes identiques, tracent leurs diagonales parallèles en contrepoint de celle des pachydermes ; dans le second, celle-ci s'est brisée à angle droit, les animaux sont comme emportés à contre-courant d'un méandre impétueux d'autobus à hue et à dia entre blocages et échappées, frottements de cuir gris contre un tollé de jaune et de rouge) et intègre, par cette même sensibilité à la musicalité chorégraphiant un espace-temps, elle-même mêlée aux sons diégétiques - klaxons, interpellations, moteurs, sonnettes de vélo comme ramassés dans le corps vibratoire de la musique, le défilé des éléphants en constante harmonisation avec le reste, et non comme élément perturbateur. Lorsque surgissent les bêtes, la musique a l'air d'être venue à leur rencontre par la gauche, dans le sens de déroulement de la pellicule : depuis le début du film, c'est vers eux qu'elle avançait, apparaissant ponctuellement dans un plan ou une séquence, s'y rechargeant, la quittant bientôt, comme si le cinéaste allait chaque fois s'assurer de sa progression vers le point de rencontre attendu. Ainsi la musique charrie tout un archipel de fragments de cette région qu'elle traverse en image, de moments qu'elle emporte dans son sillage, comme les éléphants portent chacun une part - réelle ou fantasmée, réelle et fantasmée - de l'Inde sur leur dos - leur rencontre faisant cristalliser l'événement-Kerala dans un plan toujours déjà épuisé, et par là même inépuisable.

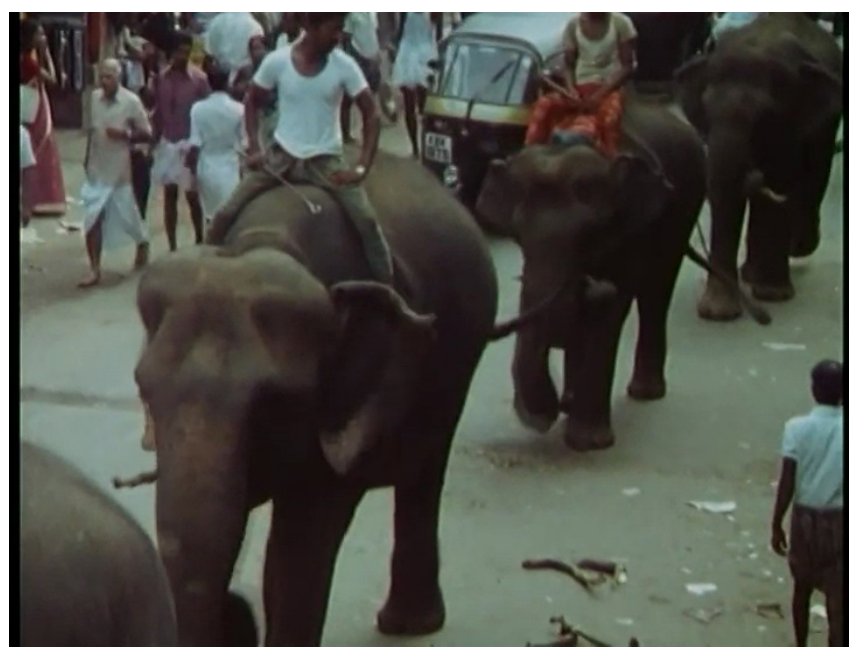

Extrait vidéo 12 : Johan van der Keuken, L'œil au-dessus du puits (1988), Entrent les éléphants, 01:01:10-01:02:14 (C) Arte France Développement, Idéale Audience International, Pieter van Huystee Film, 2006.

$6 \quad$ Voici comment van der Keuken décrit son travail sur cette séquence : " Nosh était totalement fascinée par les éléphants qu'on trouve aux endroits les plus inattendus. Nous étions dans un marché et une douzaine de ces bêtes venaient pesamment en se balançant. Or ce n'étaient pas les éléphants d'un temple mais ceux d'un cirque et on les conduisait par cette rue chaque matin à dix heures. Le lendemain nous nous sommes postés sur un avant-toit pour les filmer. J'ai bien aimé filmer l'activité de la rue où rien de particulier ne se passe et où peu à peu un éléphant se glisse dans le cadre. Magnifique ! mais en même temps je pensais : Où vais-je mettre ça ? » Je savais que ce serait tard dans le film sinon ce ne serait qu'une image exotique. Quand le spectateur est déjà en contact avec l'univers du film, cela devient possible. Différer certaines choses est une manière d'aller contre l'exotisme » (Van der Keuken 1998, p. 174). 
Si «toucher le réel » revient à demander à la musique comment toucher " toucher-l'oiseau », comment toucher " danser-l'éléphant ", alors le cinéma nous apprend que la musique est ce qui fait du réel une question, en mettant l'image à la question. Qu'est-ce qu'un oiseau? Un oiseau valse, perché sur les doigts d'un pianiste, entre une forme morte bourrée de paille et un cœur ailé battant sous les petites mains d'une aveugle. Qu'est-ce qu'un éléphant? Six ou sept éléphants passent, funambulant sur une corde de sitar, entre l'imagerie pittoresque d'une Inde de carte postale, et la vision sacrée recouvrant le gagne-pain quotidien, l'apparition animale qui remue l'animal dans le filmeur hollandais comme dans le cycliste indien, la surprise qui, même répétée chaque jour à la même heure, peut rincer d'un peu d'eau pure l'œil le plus aguerri comme la mémoire la plus colonisatrice. " D'ailleurs, si vous avez remarqué sur un magnétophone, il y a d'abord une tête d'effacement, puis une tête d'enregistrement et enfin une tête de lecture " (Paranthoën 2002, p. 37). Le montage musico-visuel pratiqué par van der Keuken nous semble rendre compte d'un trajet de la musique dans l'image similaire à celui que décrit Yann Paranthoën dans le très bel ouvrage où il relate son expérience de " tailleur de son " : s'efforçant de procéder à l'effacement d'un savoir préexistant fondé principalement sur une représentation visuelle surdéterminée par les discours dominants, la musique du film se fait enregistrement de la musique perçue dans l'existence et, elle-même ainsi taillée par sa rencontre avec l'événement, se taille un chemin dans le vif de l'image pour en produire chaque fois une lecture vivifiante. C'est penser la musique comme une anti-mémoire - cette dernière officiant toujours comme bras droit du pouvoir d'autant plus lorsque celle-ci s'épuise en faisant claquer les limites du système tonal, de l'harmonie ou du langage, et se saborde comme structure de mémorisation. Ainsi, dans L'ceil au-dessus du puits, la répétition du fragment de musique indienne que nous avons évoqué, participant chaque fois d'un agencement différent, produit ses propres différences. Elle ne joue pas le rôle de refrain, de motif mémorable et structurant, mais celui d'agent d'un processus interne d'ébranlement de l'image. "Le musicien peut dire par excellence : "Je hais la mémoire, je hais le souvenir", et cela parce qu'il affirme la puissance du devenir » (Deleuze et Guattari 1980, p. 363). La représentation, dans la relation audio-visuelle, cesse d'être une collection de memorabilia épinglés une fois pour toutes dans un album de voyages - 1'oiseau empaillé, l'éléphant sur la carte postale ; elle défait les discours visuels étalant le réel comme un éventail de modalités du plus au moins d'adéquation à une norme, pour rendre l'événement comme une expérience musicale, diffuse, complexe, irréductible " au » réel entendu comme essence - une expérience qui substitue à l'image comme réponse aux mots d'ordre du pouvoir, l'image comme question assénée à celui-ci. La musique bande alors de toute sa puissance la charge éthique et politique de l'image lorsque la question devient : un aveugle a-t-il un corps ? La scène de mise en voix de la course automobile par le jeune Herman provoque littéralement une mise en jeu des normes politico-sociales de la représentation, où la musique opère comme puissant agent de déformation. Loin de chercher à forcer le visage ou le corps de l'enfant aveugle à entrer dans le moule de la forma, la belle forme définie par les critères de l'homme blanc athlétique et symétrique, à la mécanique impeccable et au maintien bienséant, van der Keuken en accuse, par le tact de son écoute audio-visuelle, l'aspect 
monstrueux, la bouche gargantuesque, le gosier vrombissant ${ }^{7}$, les yeux laiteux, le visage mur blanc-trou blanc qui inquiète en gros plan le visage "mur blanc-trou noir" (ibid., p. 205) ${ }^{8}$ dans lequel l'Occident complaisamment se mire. Un enfant aveugle pousse le hurlement qui affole tout le monde ? Van der Keuken nous apprend qu'au cinéma, la musique peut devenir cette "violence du regard " (Van der Keuken 1998, p. 12) revendiquée par le cinéaste, qui crève les visages que la norme colle comme autant de masques sur la tête d'un aveugle, qui fait - œil pour œil, poing pour poing - d'un enfant aveugle un événement singulier rapportable à aucun tableau de classification, une tête d'effacement gueulant plein gaz dans une boîte pour faire voler en éclats de voix toute définition. Un enfant aveugle, étranger à tout souci concernant son image, à toute contrainte sociale d'auto-surveillance de ses gestes et de ses traits, balance son corps d'avant en arrière comme un « débile menta $1^{9}$ " ? Van der Keuken, en captant la prouesse vocale, le génie improvisateur de bouts de ficelle, la " puissance du devenir » (Deleuze et Guattari 1980, p. 363) de la musique ainsi expérimentée, remet en jeu les postures clichés de l'autisme pour en faire l'expérience du corps musicien, bien loin des exigences de bonne tenue qui vident les salles de concert de certains de leurs plus grands interprètes, et réduisent ces derniers à des phénomènes de foire. Aussi bien, l'écoute s'emporte elle-même dans l'épuisement de ses clichés comme de ses limites, par le jeu de la relation audio-visuelle : on n'ironise pas sur quelque chose sans s'ironiser soi-même. Ainsi, le bien entendre a tôt fait d'être aussi dictatorial que le bien voir, comme le montre Le nouvel âge glaciaire (1974) où une famille de malentendants finit, elle aussi, par être réduite à une bande de "demeurés ». Si selon la norme linguistique entendre, c'est comprendre, alors le malentendant comprend mal, le sourd ne comprend rien. Mais un sourd est-il pour autant incapable de produire de la musique ? S'il machine un bouche-doigts-micro, n'en sortira-t-il pas une course automobile? S'il expire dans une flûte, ne fera-t-il pas naître et mourir le vent dans les Andes?

\section{TENIR LE PASSAGE : LE CHANT DES MERVEILLES}

À propos de l'usage si particulier de la voix off qu'il relève dans les films d'Alexandre Sokourov, Stéphane Breton dit du cinéaste qu'il

donne une légère réverbération à sa voix pour lui faire habiter un lieu que devra rejoindre le spectateur, le lieu de l'âme, pour le dire ainsi, puisque l'âme du violon, c'est la pièce qui fait résonner ensemble la table et le dos (Breton 2010, p. 64).

C'est en ce sens que nous pouvons dire que la musique, épuisant l'image, la tordant, l'essorant, lui fait rendre l'âme des choses - non pas en un sens spirituel, comme une

7 On retrouve une telle figure dans le bombardon de Cuivres débridés, à la rencontre du swing.

8 Le chapitre 7 "Année zéro - visagéité » (Deleuze et Guattari 1980, p. 205-234) déplie le concept de visagéité en faisant du visage, "système mur blanc-trou noir » (ibid., p. 205), le signifiant sur lequel repose la sémiotique de «l'Homme blanc moyen » et du capitalisme.

9 Au début du film, Herman raconte que c'est le premier diagnostic que l'on a fait de son comportement lorsqu'il était tout jeune - notamment parce qu'il faisait des bruits. 
essence détachée de la substance du corps, renvoyant au ciel des Idées auxquelles seules l'oreille absolue et la musique comme expression parfaite donneraient accès. Le cinéma de van der Keuken nous montre et nous fait entendre une musique en souffrance de corps qui la prennent en charge et auxquels elle se recharge, cherchant en eux le résonateur capable de leur faire rendre gorge, de restituer " entre la table et le dos » leur propre musique interne qui emporte à son tour dans son épuisement les représentations et discours qui en obstruaient l'expression. Alors se compose une chaîne de corps prenant chacun à son tour le relais de la musique, une ligne de merveillance où les corps se constituent en merveilles, comme dans une séquence magnifique de Vacances prolongées filmée au Burkina Faso, aux abords puis au fil de l'eau, au confluent du Niger et du Bani.

C'est une large piste de terre dénudée traversant un paysage rythmé ça et là de quelques arbres et de maisons basses. En bordure, un grand panneau se dresse, scandant son message public: "Prudence. Lutte contre le SIDA ". La caméra y accroche son regard, joue de cette structure statique comme d'un principe dynamique qui, tantôt en avant, tantôt en arrière-plan, dirigerait les entrées et sorties des figures qui animent l'ensemble en retour: au loin, saisie de profil, passe une mobylette suivie de trois ou quatre piétons, petites silhouettes progressant au centre de l'image, comme sous le niveau du panneau masquant l'horizon ; de plus près, en buste, deux femmes à pied vêtues de tissus colorés, dont l'une porte un récipient sur la tête - on suit leur avancée de gauche à droite, de trois quarts, pour déboucher de nouveau sur le panneau, à l'arrière cette fois, de l'autre côté de la route ; en file, des hommes montés sur des ânes sont repris sous le même angle que la mobylette, le haut de leur crâne rasant le dessous du panneau qu'ils semblent franchir comme une porte, parcourant l'image de droite à gauche. Alors, on abandonne le panneau pour aller derrière un autre petit groupe à dos d'âne, même allure, variation de couleurs, démarche marquée par l'enfilade des arbres de part et d'autre de la piste, comme l'est, dans le plan suivant, celle de cette personne élancée qui va portant sur la tête de grandes paillasses. Le décor change ; arrivent nonchalamment, de face, les dromadaires. Suivent plusieurs plans tâchant d'emboîter les mouvements de divers groupes de porteurs de sacs de toile, de faisceaux de longues, longues tiges de paille, de plats en fer-blanc - nouvelle variation de tissus bariolés - se rendant manifestement au marché. Depuis le début de la séquence, une musique africaine extra-diégétique mariait son tempo soutenu et ses voix chaudes, ses tonalités pleines d'allant, aux ocres et aux verts roussis du paysage, à la montée soudaine d'un pleur d'enfant qui se fondait alors dans la modulation du chant, au pelage brun ou blanc des bêtes, les percussions se faufilant entre les foulées des ânes, suivant en léger décalage leur allure cadencée, au pas, au petit trot. Le rythme régulier accusait le jeu des lignes horizontales, verticales, diagonales de l'image, la mélodie des cordes improvisait avec les teintes multicolores des vêtements. À ce moment-là, comme de nouveau les dromadaires, montés de bédouins, passent en caravane en dessinant une courbe qu'accompagne un geste fluide de la caméra, tout ce complexe musical s'est tendu et se ramasse dans l'extinction de sa composante extra-diégétique pour se recomposer sur cette courbe en voix de fond, basse des sabots, cliquetis des harnais. À quelques plans de là, après une scène de marché formant une sorte d'intermezzo où le brouhaha des palabres, 
des négociations et des bêlements a pris le relais chaotique de la musique ; puis un trajet en voiture terminé en catastrophe par une panne-mouvements suspendus, sons raréfiés, temps perdu -, la séquence semble se rouvrir depuis le milieu du fleuve où van der Keuken filme, debout sur une pirogue. Nouvelle impulsion : un piroguier lance à la ronde, pour la caméra, pour lui-même et pour le monde, son bras qui tourne en l'air en scandant une musique à mi-chemin du chant et de la respiration. La caméra le laisse alors s'éloigner pour s'arrimer au ballet que forme le chassé-croisé d'autres pirogues, regardant l'une filer tandis que la chanson reprend hors-champ, s'attachant un moment à l'autre, remplie de femmes qui joyeusement se mettent à taper des mains, danser, chanter et rire pour divertir le filmeur : la musique a repris du corps et de la voix et peut déboucher sur une brève intervention de van der Keuken en voix off, évoquant son rapport au "fleuve humain ». Le temps semble s'étirer tandis que le soleil descend plus bas sur les embarcations, qui glissent plus lentement, donnant à la séquence un infléchissement mélancolique ténu. Les voix se sont de nouveau presque tues pour prendre les inflexions de la rumeur du fleuve, clapotements, effort des rames. Au fuselé des pirogues répond alors le ruban d'un troupeau de zébus qui fendent le fleuve en serpentant, ne laissant émerger que les cornes, la bosse et le bout du museau. Un zoom nous rapproche de la tête des bêtes et l'on entend le fleuve respirer de leur gros souffle de naseaux, la caméra tâche de suivre leur nage au plus près, et c'est bientôt le moment où elles touchent enfin la rive, remontent comme une nappe de lait qui déborde du feu ou une langue de lave éructée d'un volcan : ils sont soudain toute une légion à émerger de l'eau et l'on s'étonne de voir ces grands corps surgir comme si la matière vivante doublait, triplait, naissait de l'agitation des flots - on a l'impression d'une cosmogonie qui se rejoue lentement, avec une majesté tranquille. Qu'est-ce qui s'est passé ? Le corps ondulant des zébus, relayant la musique passée d'abord par la marche à pied ou à dos d'âne entre les pattes du grand panneau, le défilé des couleurs, le déhanché des porteurs, la course aux longues jambes des dromadaires, puis le brouhaha du marché, l'extinction du moteur, puis le chant de bras du piroguier, les rires et battements de mains des femmes, les accents émus du filmeur, les variations d'intensité de la lumière, le relâchement fatigué des rames ; ce corps monstrueux fait de cent bêtes à cornes s'est épuisé dans l'eau, soufflant, haletant, labourant le fleuve, jouant du fleuve qui s'est mis à résonner lui-même de la musique impétueuse des bêtes, comme gros d'une nouvelle origine du monde, jusqu'à dégorger cette prolifération catastrophique de corps vibrant d'une seconde naissance (extrait vidéo 13$)^{10}$. 
Extrait vidéo 13 : Johan van der Keuken, Vacances prolongées, Chanteur improvisé, pirogues, zébus : le petit monde du fleuve se relaie pour en faire lever la rumeur, 01:15:04-01:18:55 @ Arte France Développement, Idéale Audience International, Pieter van Huystee Film, 2007.

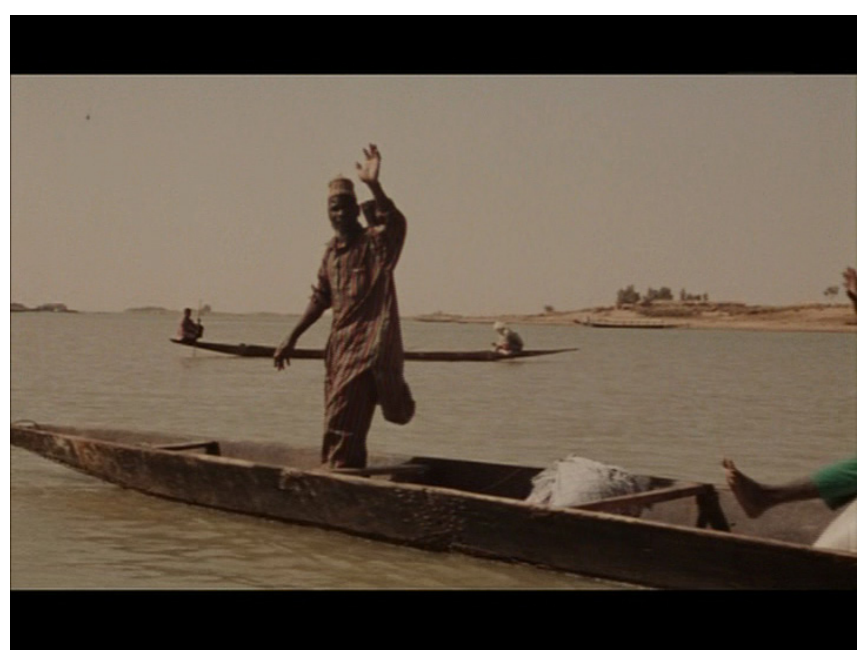

Chaque corps de cette longue chaîne musicale est devenu merveille d'avoir été saisi s'appareillant, comme Herman avec sa boîte de conserve - qui avec une pirogue, qui avec le fleuve, qui avec un dromadaire, qui avec une étoffe rose vif, qui avec un plat en fer-blanc en équilibre sur la tête, donnant à la cambrure de sa nuque une intensité comparable à celle d'une note tenue par un saxophone à bout de souffle, comme on l'entendra à la toute fin du film - pour se faire entendre comme variation continue d'un thème comme tel insaisissable. Les merveilles sont, à la Renaissance, ces objets hétéroclites, insolites, naturels ou fabriqués, collectionnés et montrés pour la fascination qu'ils provoquent, que celle-ci dénote l'admiration ou l'horreur : deux faces indissociables des mirabilia qui se caractérisent notamment par l'hybridation et la monstruosité. Le cinéma de van der Keuken retravaille musicalement cette notion de merveille à même les corps en les faisant déborder des représentations dans lesquelles ils sont cantonnés, comme nous l'avons vu avec l'enfant aveugle, comme nous le voyons encore dans cette séquence où les corps viennent au monde comme perçus pour la première fois, réinterprétés dans leur libre interprétation du milieu dans lequel ils évoluent, emboîtant le tempo d'une certaine région du monde vibrant de la rencontre de deux fleuves. En cela, la séquence s'oppose aux précédentes qui réactivent a contrario certains processus historiques d'emprisonnement dans des discours colonialistes et racistes : les corps noirs comme chair à canon en temps de guerre dans le système colonial (scène des griots se remémorant autour du feu les violences de l'armée française), ou comme systématiquement suspects de délinquance (scène des enfants sommés de dire leur prénom en gros plan contre un mur comme lors d'une interpellation policière ; leur visage barbouillé de terre et morveux ne fait qu'ajouter une couche sale à l'imagerie douteuse que fait émaner le dispositif filmique). Mais même ces plans-là génèrent leurs propres fissures par où laisser fuir les corps : ce que capte van der Keuken dans la scène nocturne des griots, c'est, en-deçà du contenu des histoires, le chant qui se compose dans l'agencement des exclamations des auditeurs ponctuant régulièrement les propos du diseur, et du crépitement visuel autant que sonore - des flammes ; tandis que les plans frontaux, agressifs, des enfants sont montés avec ceux de femmes aux pilons, dont le vif martèlement mêlé au cliquetis des coquillages décorant leurs tresses entraînées dans la scansion de leurs bras semble ouvrir des craquelures dans le mur contre lequel sont acculés les 
enfants, comme autant de brèches par lesquelles se couler. Ainsi, alors même que les représentations stigmatisantes sont mises au jour, la merveille perd son caractère figé de créature épinglée, tout en produisant, par la puissance inouïe de l'affleurement musical, sa radicale singularité.

La musique comme puissance d'émerveillement serait peut-être, au fond, l'ultime question posée à l'existence, celle du passage - de la vie à la mort et du néant à la vie telle que van der Keuken la formule dans Les vacances du cinéaste (1974) à l'occasion de la mort de son ami Ben Webster qu'il met en perspective avec la vie débordante de sa femme et de ses enfants, elle-même confrontée à la maladie du vieil homme chez qui ils passent leurs vacances, et à l'agonie du monde rural. Dans Amsterdam Global Village, un petit morceau de flûte andine faisait surgir un bébé comme par magie, ainsi que le décrit le cinéaste :

Dans l'appartement de Roberto, le Bolivien d'Amsterdam Global Village, nous avions regardé des films vidéo sur son pays. J'avais alors remarqué le côté très propre, néerlandais, de l'appartement, et tous les objets qui signalaient l'arrivée d'un bébé ; ainsi que quelques éléments qui étaient à lui - la flûte indienne accrochée au mur, par exemple. Je me suis dit qu'il fallait créer une ellipse, et faire arriver le bébé directement dans cet univers. J'ai eu la chance de pouvoir tourner quasiment le même plan de la petite baignoire, avec la même lumière de rêve, mais avec le bébé soudainement présent. C'est une figure de construction propre à la grande tradition du cinéma de fiction, dont j'entrevois la possibilité pendant le tournage. J'étais content de pouvoir poser le film dans cette tradition, et de réussir cette transition des objets à un nouvel être vivant (Van der Keuken 1998, p. 20-21).

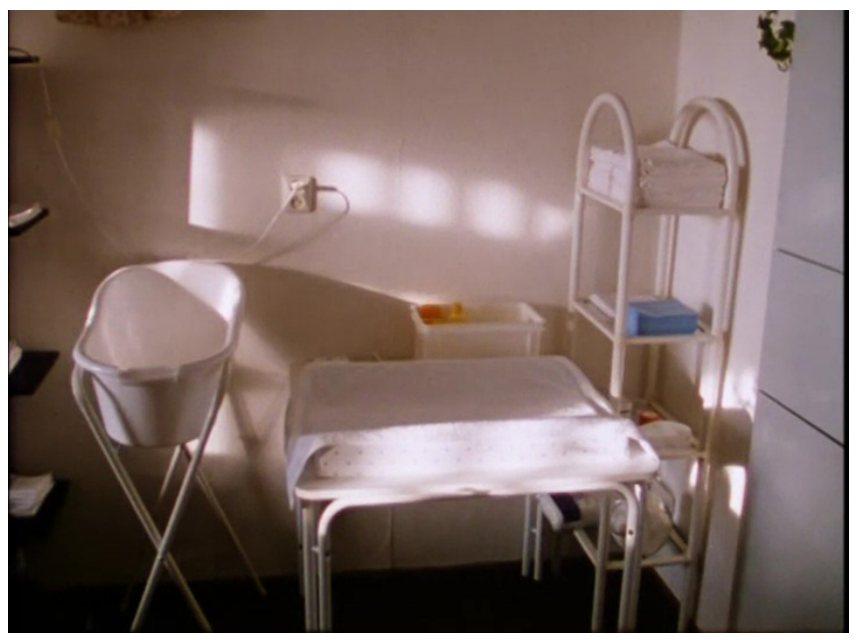

Extrait vidéo 14 : Johan van der Keuken, Amsterdam Global Village (1998), Un bébé naît soudain d'un petit air de flûte, 11:20-12:25 @ Arte France Développement, Idéale Audience International, Pieter van Huystee Flm, 2006.

Une fois de plus, l'air de musique bolivienne a épuisé la tendance des images à réduire chaque membre du couple à ses clichés - la figurine de lama ou la flûte à plumes poussant Roberto dans ses retranchements d'immigré bolivien, l'appartement tout net et bien rangé renvoyant son épouse à la bonne ménagère hollandaise - rabattant les stéréotypes l'un sur l'autre, désamorçant la charge sociale explosive qui guettait le bébé dès avant sa naissance : portée par la musique dans la "lumière de rêve ", la «transition des objets à un nouvel être vivant» a pu s'effectuer au détriment des premiers, jetés avec l'eau du bain, tandis que le bébé passait au monde non comme la somme 
des clichés boliviens et hollandais, mais comme le passage lui-même, l'événementnaissance vagissant - la musique merveillante comme "puissance du devenir ». Mais c'est dans la séquence finale du dernier film que le cinéaste rend audible le plus inaudible. Tout au long de ses "vacances prolongées ", van der Keuken, condamné par la maladie, parcourant le monde à bout de souffle dans un ultime effort pour étirer le sursis, passe et repasse en d'interminables travellings sur les paysages, déplissant la peau du monde pour lui faire expirer sa musique sous-jacente. Il cherche à faire lever, dans le déroulé d'une large piste de terre se liquéfiant en "fleuve humain ", comme dans les brumes flottant entre les pics de l'Himalaya, le chant de sa mourance. Au terme du film de sa course, le corps mourant est merveille pour avoir joué musicalement le temps qui lui reste à souffler, à souffrir, à passer, à repasser, s'épuisant à se saisir comme tel, c'est-à-dire se tenant dans le passage entre la vie et la mort, lieu de la merveille s'il en est par son caractère monstrueux, à double face : celle de l'arrêt de mort, qui met un terme au mouvement et demeure inaccessible au sentir ; celle de sa contreeffectuation, reprise créatrice sur le mode de l'interprétation musicale, qui cherche les frottements de l'impulsion pour embrasser la catastrophe. Alors seulement peut-être, " toucher le réel ", c'est faire tenir le passage, et celui-ci tient dans la rencontre de l'épuisement répété des souffles d'un saxophone et du glissando incessant des barges sur l'Amstel, à la tombée du jour toujours recommencée (extrait vidéo 15).

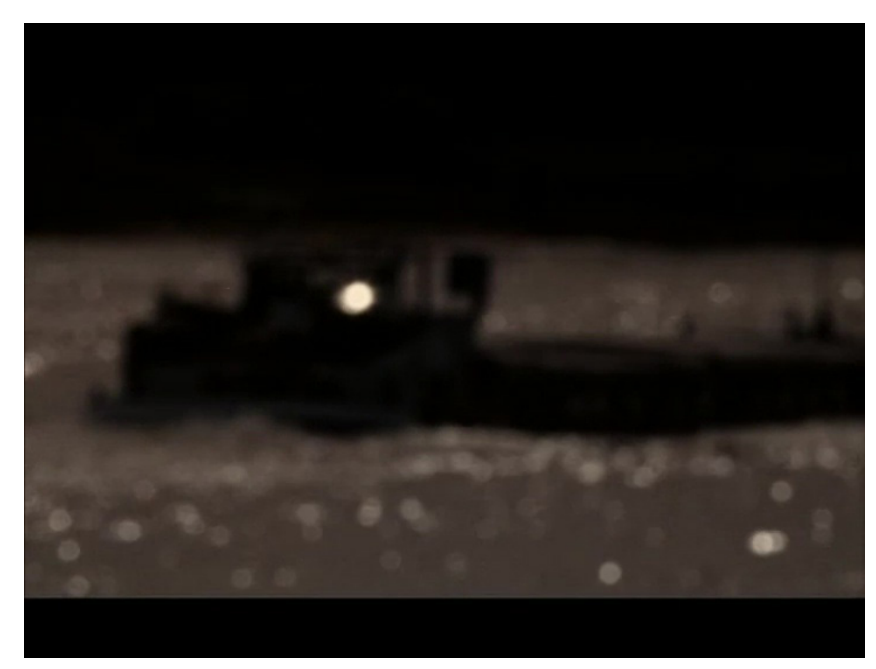

Extrait vidéo 15 : Johan van der Keuken, Vacances prolongées, Derniers feux du jour sur l'Amstel, des barges se retirent, un saxophone s'éteint, 02:15:00-02:17:23 (C) Arte France Développement, Idéale Audience International, Pieter van Huystee Film, 2007.

\section{BiBLIOGRAPHIE}

Badiou, Alain (2010), Cinéma, Paris, Nova éditions.

Barthes, Roland (1975), " Rasch », dans Julia Kristeva, Jean-Claude Milner, Nicolas Ruwet (dir.), Langue, discours, société : pour Emile Benveniste, Paris, Éditions du Seuil, p. 217-228.

Breton, Stéphane (2010), "Documentaire, l'échange de regards. Entretien avec Stéphane Breton », Esprit, n 8, p. 62-77. 
Deleuze, Gilles et Félix Guattari (1980), Mille plateaux : capitalisme et schizophrénie 2, Paris, Minuit. Paranthoën, Yann (2002), Propos d'un tailleur de son, Arles, Phonurgia nova éditions.

Van der Keuken, Johan (1998), Aventures d'un regard : films, photos, textes, Paris, Cahiers du cinéma.

Van der Keuken, Johan (2006-2008), Johan van der Keuken, édition intégrale, vol. 1-5, Paris, Arte France Développement, Idéale Audience International, Pieter van Huystee Film. 